\title{
Intergenerational Contradictions in Body Image Standards Among Australian Immigrant Mothers and Children: A Mixed-Methods Study
}

\author{
Tehzeeb Zulfiqar ${ }^{1}$, Catherine D’Este ${ }^{1}$, Lyndall Strazdins ${ }^{1} \&$ Cathy Banwell $^{1}$ \\ ${ }^{1}$ National Centre for Epidemiology \& Population Health, Research School of Population Health, ANU College of \\ Health and Medicine, The Australian National University, Australia \\ Correspondence: Tehzeeb Zulfiqar, National Centre of Epidemiology and population health, Research school of \\ population health, Australian National University, Canberra, ACT, 2601, Australia. Tel: 61-45-221-9511. E-mail: \\ Tehzeeb.zulfiqar@anu.edu.au
}

Received: February 26, 2020 Accepted: April 2, 2020 Online Published: April 13, 2020

doi:10.5539/gjhs.v12n6p47 URL: https://doi.org/10.5539/gjhs.v12n6p47

\begin{abstract}
In this mixed-methods study, we explored how gender and cultural factors, including social status were linked with children's immigrant backgrounds and their body image dissatisfaction and weight management strategies in Australia. Cross-sectional data analysis of 10-11-year-old children from the Birth cohort of the "Longitudinal Study of Australian Children" showed that approximately half of the children were dissatisfied with their body images. A higher proportion of these were children of immigrants from low-and-middle-income-countries. Additionally, about three-quarters children were actively managing their weights. Children of immigrants from low-and-middle-income-countries constituted a higher proportion of these also. Among boys, desiring a heavier body was highest for those with immigrant mothers from low-and-middle-income-countries, while the desire to be thinner was highest among girls of immigrants from low-and-middle-income-countries. Although the percentage of children who adopted strategies to gain weight was very small, boys of immigrants from low-and-middle-income-countries, in particular, were almost three times as likely as non-immigrant boys, to try to gain weight ( $18 \%$ vs $5.9 \%$, respectively).

Qualitative face-to-face interviews with immigrant mothers and their 8-11-year-old children revealed intergenerational variations in body image standards. Maternal body image standards were drawn from their origin countries, but children followed Australian norms. Despite increased obesity awareness amongst mothers, they desired higher body weight for their children, due to an association with high status and health in origin countries. However, children were aware of the stigma, unpopularity, and low status associated with high body weights in Australia. To reduce cultural and status-based obesity inequalities, Australian obesity prevention plans must include culturally responsive health promotion strategies for immigrant parents and their children to improve their knowledge about healthy weights and weight management strategies.
\end{abstract}

Keywords: Body image dissatisfaction, culture, immigrant children, intergenerational, mixed-methods, obesity.

\section{Introduction}

In Australia, where a quarter of children are either overweight or obese (henceforth referred to as overweight/obese), childhood overweight/obesity is a major public health threat (Australian Bureau of statistics, 2015). The risk of overweight/obesity is even higher in Australian-born children of immigrants from low-and-middle-Income-countries (LMICs) compared to children of Australian-born parents (Zulfiqar, Strazdins, Banwell, Dinh, \& D'Este, 2018; Zulfiqar, Strazdins, Dinh, Banwell, \& D’Este, 2018). These children of immigrants also experience higher body image dissatisfaction (BID), weight stigma, lower self-esteem and poorer mental health (Gualdi-Russo et al., 2012; Toselli, Brasili, \& Spiga, 2014), compared to children of the host population with dissimilar linguistic, culinary and cultural norms.

BID is a multidimensional psychosocial concept and expresses the "displeasure with how the body appears to oneself" (Rosen, 2013; Smolak, 2004). BID appears in children as young as five years and may result in eating disorders (Birbeck \& Drummond, 2005). Once seen largely as an issue associated with girls, more recently BID and weight concerns are also reported in boys (Jones, Bain, \& King, 2008; Klein, 2008). However, marked variations regarding BID persist between cultures, many of which have gender dimensions (Mitchison \& Mond, 2015; Mond et al., 2014; O'dea \& Caputi, 2001; Ricciardelli, McCabe, Holt, \& Finemore, 2003). In many 
high-income-countries (HICs) such as Australia, the desired body image for boys is muscular, due to its functional and instrumental qualities, in contrast to slenderness for girls, considered aesthetically appealing. However, in many LMICs with historical shortages, heavier bodies for both boys and girls are considered a sign of beauty, prosperity, and health (Gualdi-Russo et al., 2012; Renzaho, McCabe, \& Swinburn, 2012). These cultural differences in body image preferences may complicate how immigrants recognise and adopt strategies to prevent obesity in their children (Gualdi-Russo et al., 2012; Renzaho, McCabe, \& Swinburn, 2012). As children of immigrants acculturate, they adopt the norms of the host societies, which may result in intergenerational and interfamilial stress (Gualdi-Russo et al., 2012; O'dea \& Caputi, 2001; Pallan, Hiam, Duda, \& Adab, 2011; Renzaho, McCabe, \& Swinburn, 2012; Toselli et al., 2014). However, despite hosting a large multicultural immigrant population, there is little Australian evidence connecting the social and cultural influences from origin countries of immigrants to BID, and overweight/obesity risk in their children.

We used an exploratory mixed-methods design in this research where a qualitative study was conducted to contextualise the cross-sectional quantitative study findings (Van Griensven, Moore, \& Hall, 2014), and to unpack the explanatory mechanisms. First, cross-sectional data of 10-11-year-old boys and girls from Birth cohort of the "Longitudinal Study of Australian children" (LSAC) was analysed to describe BID and weight management strategies among children of immigrants and non-immigrant Australian mothers. The focus of our research was children of immigrants from LMICs. However, children of immigrants from HICs were also included in the quantitative analysis to test whether immigration differentially affects BID and weight management strategies among children by the economic development of their maternal origin countries.

Second, sociocultural factors around BID were explored through in-depth face-to-face structured interviews with mothers from three LMICs (Bangladesh, India, and Pakistan) and their 8-11-year-old children. Mother and child interviews were conducted to explore the intergenerational conflicts and contradictions regarding perceptions, and practices about child weight status. Immigrant mothers from these three countries were selected because they are the second-largest growing population of Australian skilled immigrants (Australian Institute of Health and Welfare, 2018). Immigrants from these countries are at higher risk of adult overweight/obesity and obesity-related health conditions in Australia (Pasupuleti, Jatrana, \& Richardson, 2016; WHO Expert Consultation, 2004). However, little is known about their cultural practices which may increase the risk of overweight/obesity in their children.

\section{Methods}

\subsection{Cross-Sectional Study}

The data for this cross-sectional study were drawn from the Birth cohort of LSAC which is an ongoing nationally representative cohort study, with biennial data collection (Soloff, Lawrence, \& Johnstone, 2005). The sampling frame was drawn from the Medicare Australia enrolment database, which covers all Australian permanent residents. Multistage cluster sampling, stratified by both state/territories and metropolitan/non-metropolitan areas was adopted. Approximately one in ten postcodes (the primary sampling unit) were randomly selected, with probability proportional to size (Soloff et al., 2005). Within selected, postcodes. children had an approximately equal chance (one in 25) of selection (Soloff et al., 2005).

Every two years, after obtaining informed consent, trained interviewers conducted face-to-face interviews primarily with the child's mother. However, when children were 8-9-year-old, they became primary respondents to some questions relating to general cognitive abilities, body images and weight management strategies (Daraganova, 2013). Interviewers also recorded child's anthropometrical measurements during these biennial interviews.

For this study, we conducted crosssectional data analysis of children aged 10-11-years with known immigrant backgrounds ( $\mathrm{n}=3729)$. This age was chosen because at this age significant gender differences in body image, and attitudes, and behaviours relating to body weight emerge in children (Ricciardelli \& McCabe, 2001).

\section{Variables Used in the Cross-Sectional Study}

1) Child Immigrant backgrounds were determined by maternal birth countries. This decision was based on the strong influence of mothers in determining child's nutritional status (Baker, Balistreri, \& Van Hook, 2009; Gibson et al., 2016; Gualdi-Russo et al., 2012; Hancock, Lawrence, \& Zubrick, 2014; Linabery et al., 2013; Ziauddeen, Roderick, Macklon, \& Alwan, 2018).

Children were classified as non-immigrant Australians if they were born in Australia or born-overseas with an Australian-born mother. Children were considered immigrants if they had an overseas-born mother (Australian Bureau of statistics, 2012). Children were further classified using the socioeconomic development of their maternal birth countries, using the United Nations Development Program (UNDP) Human Development Index 
(HDI) scores of 2015. Children with mothers born in HICs were classified as children from HICs backgrounds. If the mothers were born in LMICs, children were considered as children from LMICs backgrounds. LMICs included countries with HDI scores of $<0 \cdot 7$, and HICs with HDI scores of $\geq 0.7$ (UNDP, 2016).

2) Body image dissatisfaction. In the LSAC, 10-11-year-old boys and girls were shown the Pictorial Body Image Instrument (Collins, 1991). A set of seven body silhouettes were shown to children, matched to their gender (Figure 1) (Australian Institute of Family Studies, 2014). These silhouettes ranged in size from thinnest to largest, ordered morphologically. Children were asked to choose the silhouette they believed was most similar to their body image (perceived body image), as well as the silhouette they desired to be (desired body image), with scores of 1 to 7 allocated to the relevant image, as shown in Figure 1. Body image dissatisfaction (BID) was calculated by subtracting the desired body image score from the perceived body image score (Daraganova, 2013).

A positive score indicated that the perceived body image was larger than the desired body image. A negative score indicated that perceived image was smaller than the desired body image. A score of zero indicated that the child was satisfied with their body image (same body silhouette chosen as perceived and desired).

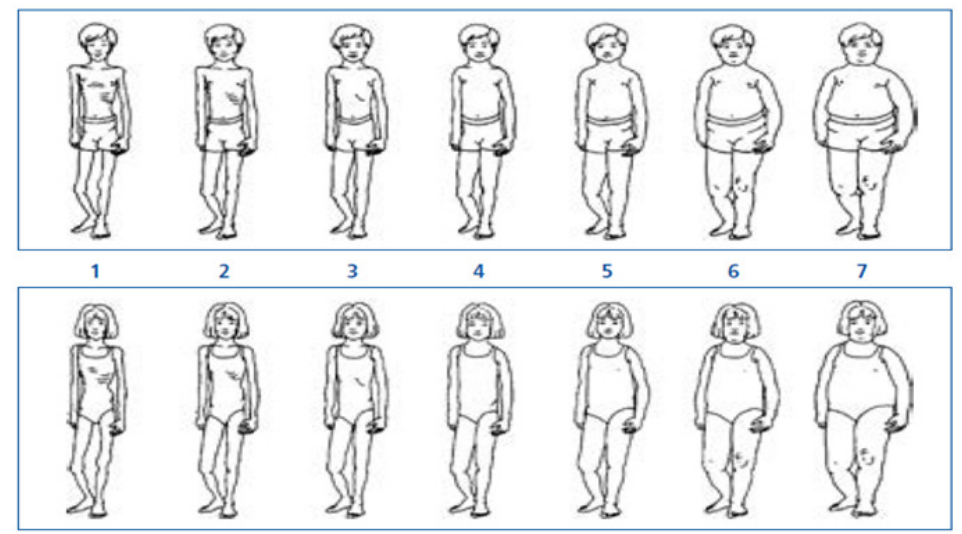

Figure 1. Colin's body silhouette chart used in LSAC and the present study

3) Weight management strategies. At age 10-11-years, boys and girls were asked whether they had done anything to control their weight (tried to lose weight or keep from gaining weight) during the last 12 months. The children were then asked to pick one option that best described what they were trying to do about their weight at the time of the interview. Children could specify whether they were trying to lose weight, gain weight, stay the same, or do nothing (Daraganova, 2013).

4) Child weight status. Body Mass Index (BMI) scores were calculated by weight (in light clothing) / height (without shoes) squared $\left(\mathrm{kg} / \mathrm{m}^{2}\right)$, measured by trained interviewers at each visit using standardized equipment (Soloff et al., 2005). In the LSAC dataset, these categories were determined by International Obesity Task Force (IOTF) age-and-gender-specific criteria. The IOTF cut-off points for overweight and obesity correspond to 25 and $30 \mathrm{~kg} / \mathrm{m}^{2}$ in young adults aged 18, which are extrapolated to children (Cole, Bellizzi, Flegal, \& Dietz, 2000).

5) Other variables used to describe the cross-sectional sample were maternal weight status based on self-reported height and weight (not overweight/obese vs overweight/obese), main language spoken at home (English/non-English), and family socioeconomic position (SEP). Family SEP summarized the social and economic capital available to families and comprised of combined annual family income, both parents' employment status and education (Blakemore, Strazdins, \& Gibbings, 2009). The summary standardised SEP scores were divided into the lowest $25 \%$, the middle $50 \%$, and the highest $25 \%$.

\subsection{Qualitative Study}

The qualitative study sample was not from the LSAC. Immigrant mothers from Bangladesh, India, or Pakistan, and their 8-11-year-old children were eligible to participate in the study if the mothers lived in Australia for at least a year, had a child aged 8-11 years, were primary meal preparers, and could communicate in English.

Snowball sampling was used as it is effective in identifying hard to reach populations in research (Sadler, Lee, Lim, 
\& Fullerton, 2010). The first mother was identified through a community contact, who later identified other mothers from her contacts and provided them the email and telephone number of the first author (TZ). On contact, TZ explained the study and followed up by mailing out the maternal and child participant information sheets. A week later, interviews were scheduled.

Face-to-face structured interviews were conducted from March 2017-September 2017, in Canberra, Australia. Interviews were conducted at the respondent's home; except in three cases where they were conducted at local cafés. Mothers and children were given the opportunity to seek clarification and to opt-out at any stage. Prior to the interviews consent forms were signed by the mothers and their children. If there was more than one child aged 8-11, the oldest child was interviewed.

In the majority of cases, the child's interview followed a maternal interview. To ensure credibility and trustworthiness of the data (Tracy, 2013), many questions in the qualitative study were adopted from the cross-sectional study. These included questions related to sociodemographic charateristics, body image and weight management strategies. The maternal and child interviews commenced by asking them separately to rate the child's body image (perceived and ideal) from the same "Pictorial Body Image Instrument" (Collins, 1991), used in the LSAC. Children were then asked whether they had adopted any weight management stratgies during the last 12 months and at the time of the interview.

An interview guide was used to steer the discussion around body image and weight with mothers and children. This gave the flexibility to adjust the questions during the interview (Tracy, 2013). Maternal interviews lasted for an hour and were conducted without the child present. The child's interview (with the mother present), took approximately twenty minutes. Mothers received AU\$15 supermarket voucher to compensate for their time.

\subsection{Ethical Approval}

The LSAC is approved by the Australian Institute of Family Studies Ethics Committee. The cross-sectional and qualitative study presented in this paper was approved by the Australian National University Human Research Ethics Committee (Protocol No. 2015/421 and 2016/473, respectively).

\section{Data Analysis}

\subsection{Cross-Sectional Data Analysis}

Cross-sectional data analysis of 10-11-year-old children from LSAC was conducted in Stata 15.0. Statistical significance was set at $\mathrm{p}<0.05$. Analyses accounted for the sampling design, including the use of sampling weights incorporating non-response and post-stratification.

Child body images were broadly categorised to thin bodies (picture 1,2 and 3), average bodies (picture 4) and heavy bodies (picture 5,6, and 7). BID was categorised into those 1) who were satisfied with body weight (no difference between perceived and desired body image), who were unsatisfied and wanted a heavier body image (negative BID score) and who were unsatisfied and wanted a thinner body image (positive BID score).

Child actual weight status was classified as underweight, normal weight and overweight/obese (Cole et al., 2000). The decision to combine overweight/obesity as a single category was due to small numbers of children of immigrants from LMICs and because of our previous analysis where we found that these two groups shared similar risk-factors at every age (Zulfiqar et al., 2018). Due to the evidence of distinct gender-specific variations in body images, cross-sectional data analysis was conducted separately for boys and girls.

We present descriptive characteristics (frequencies and percentages) of 3729 children by their immigrant backgrounds, and compare them using the Chi-squared test. The association between BID and the use of weight management strategies was examined separately for boys and girls and compared by their immigrant status using the Chi-squared test. The proportion of children by their BID category and their weight management strategies are presented graphically in the body of the paper, and by frequencies and percentages in supplementary tables.

\subsection{Qualitative Data Analysis}

Descriptive characteristics of mothers and children from the qualitative interviews were recorded and analysed in Excel v2016. BMI were calculated from maternal reported height and weight for themselves and their children. Questions related to BID and weight management strategies were used to steer the structured interviews with mothers and children.

The first three maternal and children interviews were transcribed by TZ. The remainder of the interviews were transcribed by a transcription agency (www.rev.com). Each transcript was then matched and checked with the recorded interviews for accuracy by TZ. ATLAS.ti (version 8) was used to manage, code, and analyse the data. 
Thematic analysis was conducted to capture important themes and patterns relating to the research questions (Braun, Clarke, \& Terry, 2014). TZ developed initial codes based on interview questions and a literature review. These codes were refined and new codes and concepts were added from transcripts (Saldaña, 2015). Broad themes were identified by the method of constant comparison, accounting for similarities and differences among mothers and children (Saldaña, 2015). Maternal and child interviews were analysed separately and then compared to understand similarities and differences across generations. Transcripts were also shared with the mothers for comments and for accuracy of responses.

\section{Results}

\subsection{Cross-Sectional Study}

The cross-sectional sample from LSAC comprised of 2993 (80\%) 10-11-year-old non-immigrant Australian children, $383(11 \%)$ children from HICs and $353(9 \%)$ children from LMICs backgrounds (Table 1). Non-immigrant Australian children also included 61 (1.6\%) Aboriginal and Torres Strait Islanders. At 10-11 years, a slightly higher percentage of boys (34\%) and girls (29\%) from LMICs were overweight/obese compared to boys (23\%) and girls (22\%) from HICs, and boys and girls from Australian backgrounds ( $25 \%$ and $24 \%$ respectively). This association between BMI categories and child immigrant backgrounds were not statistically significant for girls $(\mathrm{p}=0.18)$ and only marginally non-significant for boys $(\mathrm{p}=0.054)$.

Table 1. Descriptive statistics of cross-sectional sample of 10-11-year-old children from Birth Cohort of the "Longitudinal Study of Australian children"

\begin{tabular}{|c|c|c|c|c|}
\hline & $\begin{array}{l}\text { Australians } \\
\mathbf{n} \% \\
\mathbf{n}=\mathbf{2 9 9 3}\end{array}$ & $\begin{array}{l}\text { HICs backgrounds } \\
\mathrm{n} \% \\
\mathrm{n}=\mathbf{3 8 3}\end{array}$ & $\begin{array}{l}\text { LMICs backgrounds } \\
\mathrm{n} \% \\
\mathrm{n}=\mathbf{3 5 3}\end{array}$ & P value \\
\hline Boys & $1529(51.1)$ & $201(52.5)$ & $180(51.0)$ & \\
\hline Girls & $1464(48.9)$ & $182(47.5)$ & $173(49.0)$ & 0.9 \\
\hline Mother not overweight or obese & $1309(45.9)$ & $208(55.6)$ & $170(54.3)$ & \\
\hline Mother overweight or obese & $1540(54.1)$ & $166(44.4)$ & $143(45.7)$ & $<0.001$ \\
\hline \multicolumn{5}{|l|}{ Main language spoken at home } \\
\hline English & $2871(95.9)$ & $318(83.0)$ & $94(26.6)$ & $<0.001$ \\
\hline Non-English & $122(4.1)$ & $65(17.0)$ & $259(73.4)$ & \\
\hline \multicolumn{5}{|l|}{ Family socio-economic position } \\
\hline Low & $758(25.8)$ & $75(19.7)$ & $104(30.2)$ & \\
\hline Middle & $1446(49.3)$ & $205(53.8)$ & $149(43.3)$ & 0.01 \\
\hline High & 731 (24.9) & $101(26.5)$ & $91(26.5)$ & \\
\hline Boys & $\mathrm{n}=1452$ & $\mathrm{n}=187$ & $\mathrm{n}=173$ & \\
\hline underweight & $91(6.3)$ & $13(6.6)$ & $15(8.9)$ & \\
\hline Normal weight & $1019(68.4)$ & $133(70.4)$ & $105(56.9)$ & 0.054 \\
\hline Overweight/obese & $342(25.3)$ & $41(23.0)$ & $53(34.1)$ & \\
\hline Girls & $\mathrm{n}=1391$ & $\mathrm{n}=181$ & $\mathrm{n}=158$ & \\
\hline underweight & $90(6.9)$ & $15(8.4)$ & $15(9.5)$ & \\
\hline Normal weight & 973 (69.9) & $127(70.2)$ & $97(61.4)$ & 0.18 \\
\hline Overweight/obese & 328 (23.6) & $39(21.5)$ & $46(29.1)$ & \\
\hline
\end{tabular}

- Abbreviations: HICs=high-income-countries, LMICs= Low-and-middle-income-countries

- Percentages are weighted and rounded.

- P-value for chi-square test, comparing variables across immigrant status (Australian, HICs \& LMICs).

- Numbers may not add due to missing values 


\section{Body Image Dissatisfaction}

BID varied significantly by child immigrant status (Figure 2, Supplementary Table 1). Approximately $45 \%$ of Australian boys and girls had BID. Compared to Australian boys, BID was significantly low in boys from HICs $(33 \%)$ and high in boys from LMICs backgrounds $(52 \% ; \mathrm{p}=0.002)$. Similarly, compared to Australian girls, BID was significantly low in girls from HICs $(39 \%)$ and high in girls from LMICs backgrounds $(56 \%$; $\mathrm{p}=0.008)$. Among boys, desiring a heavier body was highest for those with LMICs backgrounds, while desiring a thinner body was highest for girls from LMICs backgrounds.

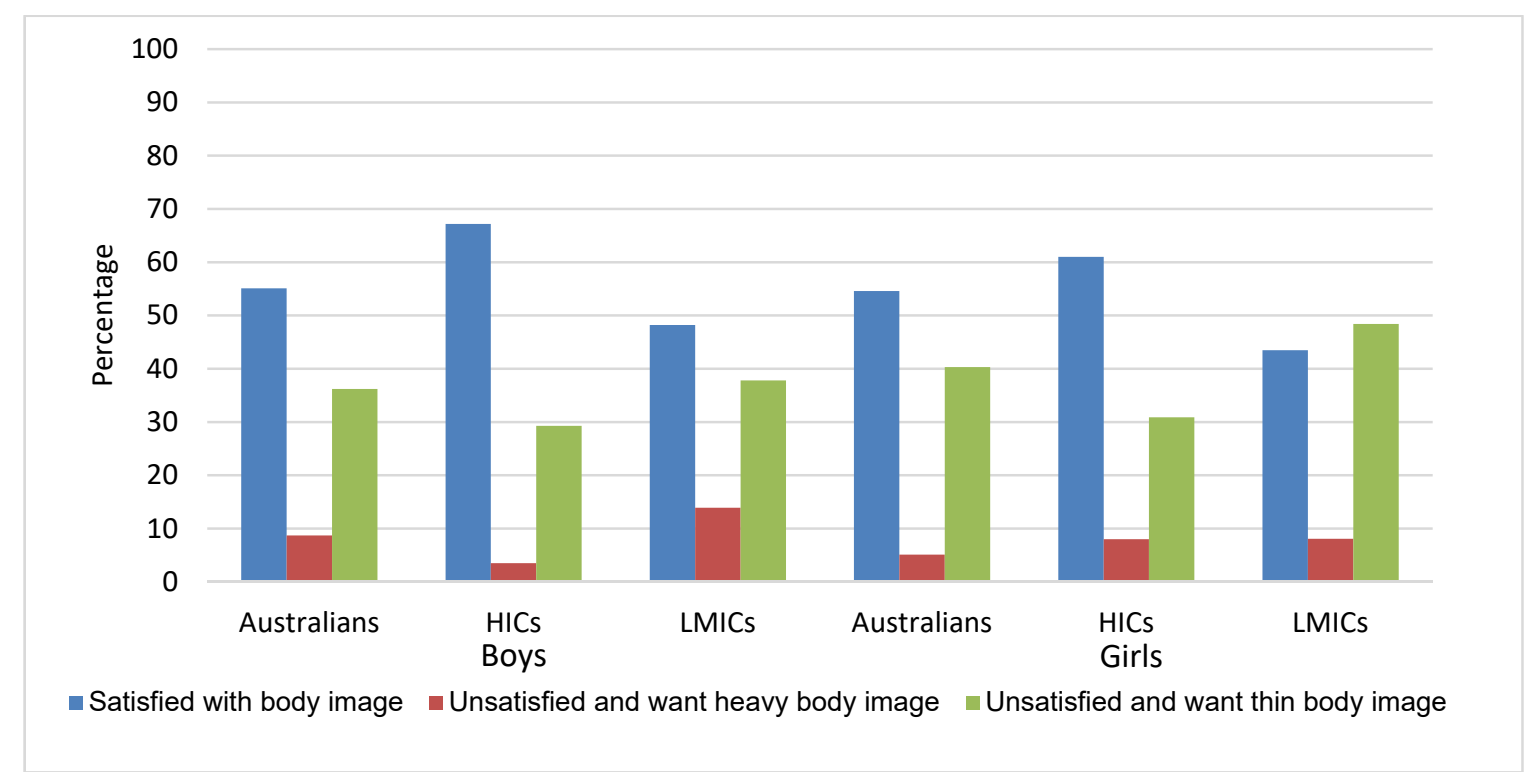

Figure 2. Body image dissatisfaction in cross-sectional sample of 10-11-year-old boys and girls from Birth Cohort of the "Longitudinal Study of Australian children" by their immigrant backgrounds

\section{Weight Management Strategies}

Approximately three quarters (2699/3548) of 10-11-year-old children adopted weight management strategies to gain, lose, or to maintain their current weight (Figure 3, Supplementary Table 2). The use of weight management strategies varied significantly by immigrant status for both boys and girls $(\mathrm{p}<0.001$ for both). Children from LMICs backgrounds had the highest percentage undertaking strategies for either weight loss or weight gain. Forty-six percent boys from LMICs, $41 \%$ from HICs and $40 \%$ from Australian backgrounds had engaged in strategies to lose weight. For girls, $45 \%$ from LMICs, $28 \%$ from HICs, and $41 \%$ from Australian backgrounds had tried to lose weight. Although the percentage of boys and girls who adopted strategies to gain weight were very small, boys from LMICs backgrounds in particular were almost three times as likely as the other two groups to try to gain weight (18\% boys from LMICs vs $5.9 \%$ boys from HICs and Australian backgrounds). 


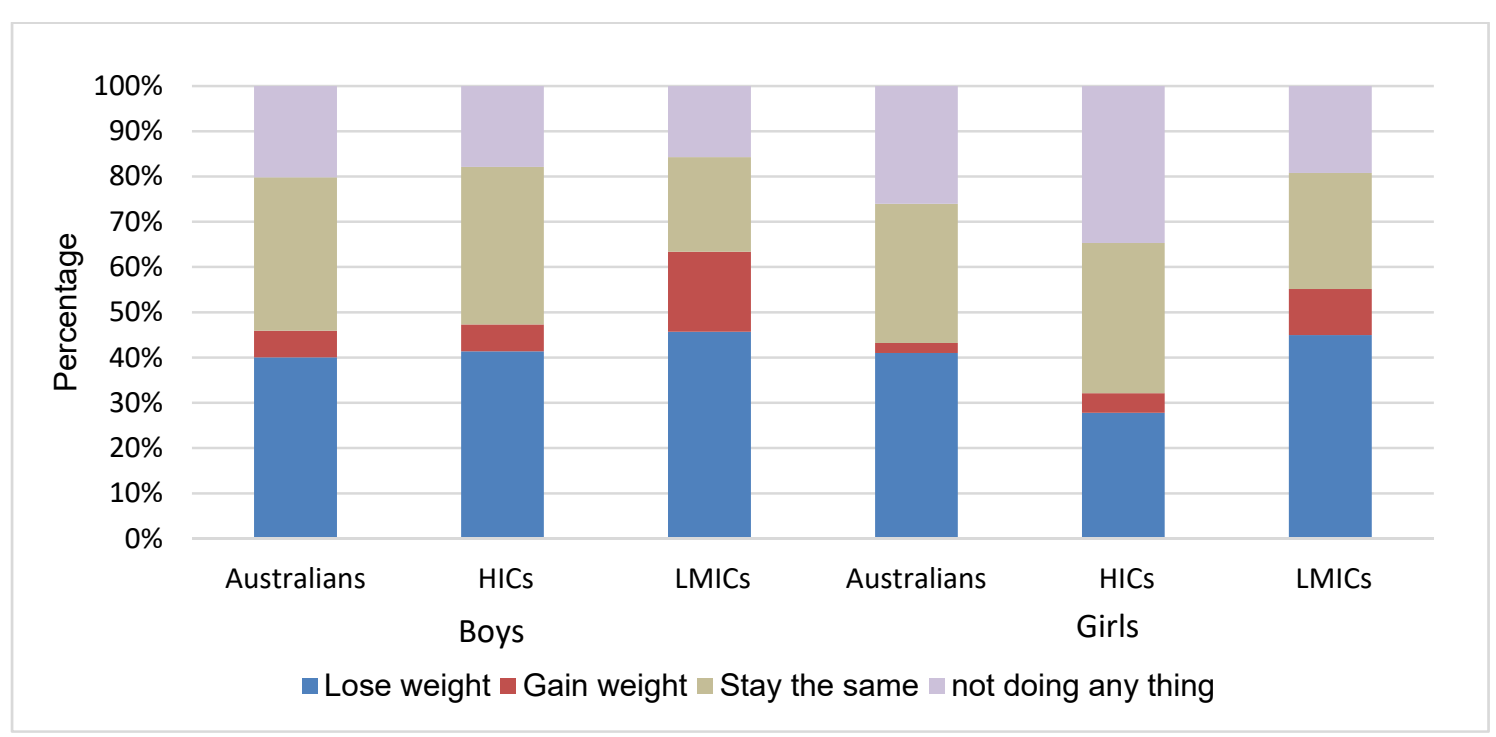

Figure 3. Percentage of 10-11-year-old boys and girls who were using weight management strategies in B Cohort of the "Longitudinal study of Australian Children"

In summary, the cross-sectional study results indicate that a disconcertingly higher proportion of children from LMICs backgrounds had BID and were trying to manage their weight. In the next section we will explore these results through narratives of mothers and their children.

\subsection{Qualitative Study}

Fourteen mothers and twelve children aged 8-10-years (Four mothers and three children from Bangladesh, five mothers and four children from India and five mothers and children from Pakistani backgrounds) were interviewed. All the mothers considered themselves as middle-class Australians but identified their origin country as their primary identity. Except one, all were fluent in English. Majority mothers (12/14) communicated with children using both English and their native language. Except four, all children were Australian-born (Table 2).

Table 2. Demographic characteristics of participants of the qualitative study

\begin{tabular}{ll}
\hline Characteristics & $\mathbf{n}(\mathbf{\%})$ \\
\hline Mothers & $\mathbf{n}=\mathbf{1 4}$ \\
\hline Age in years (mean, range) & $39(32-48)$ \\
\hline Self-identified ethnicity & $5(36)$ \\
Pakistani & $5(36)$ \\
Indian & $4(29)$ \\
Bangladeshi & $10(71)$ \\
\hline Arrived before 2009 & $4(29)$ \\
Arrived during or after 2009 & $1(7)$ \\
\hline Maternal education & $8(57)$ \\
Secondary school education & $5(36)$ \\
Bachelors & $12(86)$ \\
Masters & $2(14)$ \\
\hline Mixed (English and native) language spoken at home & $7(50)$ \\
Only native language spoken at home & $7(50)$ \\
\hline Not overweight/obese & \\
Overweight/obese & \\
\hline
\end{tabular}




\begin{tabular}{ll}
\hline Children & $\mathbf{n = 1 2}$ \\
\hline Age in years (mean, range) & $10.1(8-12)$ \\
\hline Girls & $3(25)$ \\
Boys & $9(75)$ \\
\hline Children born in Australia & $8(67)$ \\
Children born overseas & $4(33)$ \\
\hline Children having normal weight* & $10(83)$ \\
Children who are overweight/obese & $2(17)$ \\
\hline
\end{tabular}

Note. Maternal and child weight status were calculated by maternal self-report of height/weight for herself and her child, and calculating BMI as weight/height squared $\left(\mathrm{kg} / \mathrm{m}^{2}\right)$ according to IOTF classification

The main themes identified in maternal and children interviews included: 1) Influence of maternal origin culture; 2) obesity and health literacy; 3) weight management.

\section{Influence of Maternal Origin Culture}

Strong cultural and status-linked influences of the origin country were observed on maternal views of their child's body image. Mothers associated higher body images in infants and preschool-aged children as signs of good motherhood, health, and status. Thinness in younger children was stressful for mothers due to its association with diseases. Mothers reported no gender-specific variations for body image for younger children, however, for older children, there was a marked divergence.

Distinct gender-specific cultural differences emerged for preadolescent and adolescent children. The norms for adolescent girls changed to tall, and thin bodies, and for boys taller, heavier or muscular bodies were preferred. According to mothers the beauty standards for adolescent girls in their origin countries were thinness, tallness, and lighter skin tones. Mothers did not want their daughters to gain weight as "overweight girls are not marriageable". However, higher weight among males expressed strength and high status. Even for young boys, higher body weights were preferred due to the future role of boys as breadwinners for the family.

"... everybody wants their babies to be chubby, especially the mothers to get the mother-in-law off her back that she's not being a good mother. But when a girl reaches puberty, and if she is putting on weight, then mothers are saying, "Oh, you are putting on weight, why you are gaining weight." But when the boys are doing that, in general, we are okay if the boys are putting on weight. They need it"

Pakistani mother aged 38 years.

Immigrants maintained regular contact with their families in origin countries through Skype, phone, social media and frequent visits to their origin countries. While regular contact helped reduce loneliness, it also resulted in stress, particularly when a comparison of their children's weight was done with children in the origin countries. Mothers were urged to adopt strategies for children to have a "culturally appropriate body image", which was often heavier than the Australian norms. Some mothers even consulted doctors to seek advice on their children's weight due to pressure from their families. Their families in origin countries found it difficult to comprehend how children of their families in rich countries such as Australia were not fat.

When I always go back to Bangladesh and everybody sees them. The first thing they say, "Why they are so skinny?" I said, "I don't know." They say, "You don't give them much food and they live in Australia."

Bangladeshi mother aged 40.

Mothers did report a fairly recent change favouring thinness in the urban upper-middle educated class in their origin countries, which was the background of all of our mothers. Increased media discourse, rise in chronic health conditions, and frequent international travel has introduced people to global trends in diets and physical activities, and raised public perceptions on adverse health consequences of overweight/obesity. Although higher weights were still widely considered a sign of prosperity, and health among older generations, younger generations thought otherwise. Some of this change in younger generations was due to the promotion of mesomorphic (muscular, athletic and lean) male bodies and petite and delicate female bodies in Western and Indian cinema.

\section{Obesity and Health Literacy}

The mothers had arrived in Australia from countries where obesity rates and awareness of obesity was low. In their 
origin countries, obesity was generally favoured and considered a sign of good health, and social status. Although immigration to Australia with high obesity prevention discourse, increased their awareness about unhealthy bodyweights to some extent, their obesity knowledge was still sub-optimal. For example, the maternal definition of obesity was "having a big belly". In the absence of a big belly, higher body weight was considered "muscular" or "healthy". Mothers avoided using terms such as fatness or obesity and referred to children who were overweight/obese as "healthy". A consensus among mothers was that children should have some fat and not be as skinny as Australian children. Mothers thought "some weight" on children is protective against diseases and indicate that they belong to an affluent family. They also believed that "if they (children) have a good weight, then the weight converts into height". All mothers agreed that "skinny (silhouette $1 \& 2$ in Colin's chart) is poor health". A healthy child was defined as one who "eats well", "is fit", "do not have headaches", "is not pale" and "have a little more weight".

In contrast to mothers, obesity was viewed as highly stigmatised by both boys and girls who did not "want to get fat" (silhouette $6 \& 7$ in Colin's chart). These first and second-generation children of immigrants associated obesity with poor diet, low physical activities, lower status, and poor health. Children with higher weights were considered "unpopular", "lazy", and "poor in sports and studies" because they "can't play cricket or run fast", "get tired easily". Although children echoed their mother's obesity definition (big belly), but all of them thought that being "skinny is good".

Mothers and children were well aware of the dietary causes of obesity. Diet was considered as a strong determinant of obesity, after genes. Children considered fast foods and sugar as the main causes of obesity. Obese children were those who had poor self-control, who ate "too much sugar, and hamburgers". Children learned about the health implications of sugar and fast foods from their parents, television, and school teachers. Children also conveyed the knowledge gained at school regarding healthy eating and physical activities to the mothers, however, most mothers found it hard to incorporate this knowledge to their daily traditional meals, as the information was not specific to their cooking techniques and dietary habits. Mothers reported an increase in children's fruit and vegetable consumption after they started school. Children ate their traditional home-cooked meals at home, but refused to take them to school as they considered them "unpopular" and "low status". Instead, they preferred to eat canteen items at school such as pizza, meat pies, hot pasta, and spaghetti which were popular with their peers.

The benefits of physical activities for obesity prevention were known to all the mothers and their children. However, mothers did not know about the type and duration of activity levels appropriate for their children. Few (4/14) considered school-based physical activities were sufficient and discouraged out of school activities. All the mothers prioritised education, religious and language classes over sporting activities on the weekends. Children, on the other hand, wanted sports participation on weekends, as it expressed the Australian way of life. Mothers compared children's physical activities with children of origin countries, children compared their physical activities with their Australian counterparts. Therefore, mothers were satisfied with their children's physical activities, however, their children wanted to increase their physical activities to the level of their Australian counterparts. Girls in general, and immigrant girls, in particular, were considered less physically active than boys.

\section{Weight Management}

All children in our qualitative study reported active management of their weight, even if they were satisfied with their body images. Popular weight management strategies were dietary. There was less emphasis on increasing physical activities particularly amongst girls, due to religious, cultural and security reasons. Mothers reported similar patterns of weight management in their origin countries (more reliance on diet than physical activities).

Approximately two thirds (9/14) of mothers were concerned about their children weight and the majority wanted their children to gain weight. More than half of children were satisfied with their body weights and wanted to remain the same. Boys preferred slightly higher body weights as they expressed masculinity and better physical and mental health. Many wanted to be "taller "and "muscular" and wanted to join a gym to develop a muscular body. For example, one boy said "I look stronger and bigger and then I can be better at basketball".

Despite being satisfied with their body images, girls in our study were actively managing their weights on diet, due to their mother's dissatisfaction with their bodyweights. For girls the option of managing weight by increasing physical activities was not favoured by their families. This gendered divide became obvious with age as physical activities of boys increased and that of girls decreased. Adolescent girls were more sedentary and spent their free time reading books, watching television or helping their mothers in domestic chores. Child safety, security, fear of racism, and discrimination were some reasons for not allowing children to participate in out of school activities. However, this was not applied evenly across genders. 


\section{Discussion}

In this mixed method research using the representative cross-sectional sample of 10-11-year-old Australian children, we showed significant differences in BID in children from LMICs, in contrast to children from HICs backgrounds and non-immigrant Australian children, despite little variation in their body weights (BMI categories were similar across child immigrant backgrounds). BID was reflected in the weight management strategies. More boys from LMICs backgrounds wanted to be heavier and therefore were using weight gain strategies. In contrast, more girls from LMICs wanted to be thin and were using weight loss strategies.

Our structured interviews showed that higher BID among children from LMICs may be due to the difference in body image standards of immigrant parents and those common in the host country. Such contradictions in body images are likely to be stressful for children and increase unhealthy weight management strategies and risk of eating disorders (Renzaho, McCabe, \& Swinburn, 2012).

Our cross-sectional study also showed that among the children dissatisfied with their body images, majority desired to have a thinner body. Similar results were reported among children of immigrants from other countries also (Gualdi-Russo et al., 2012; Kimber, Couturier, Georgiades, Wahoush, \& Jack, 2015; Olvera, Suminski, \& Power, 2005; Pallan et al., 2011; Toselli et al., 2014; Veldhuis, te Poel, Pepping, Konijn, \& Spekman, 2017). The children who adopted weight loss strategies were also higher from LMICs backgrounds, relative to their peers, despite little difference in their body weights. Additionally, amongst the very few children who wanted to gain weight the desire for heavier body was highest amongst boys from LMICs backgrounds.

Our face-to-face structured interviews with mothers from three LMICs and their children explained patterns in the cross-sectional study and contextualised children's preoccupation with their bodyweights. Maternal accounts highlighted the influence of their origin countries on the desire for heavier bodies for children, where heavy bodies in boys represented strength, health, and prosperity (Gualdi-Russo et al., 2012; Pallan et al., 2011; Park, 2017; Renzaho, McCabe, \& Swinburn, 2012). However, for girls, the body standards were thinner than boys.

The qualitative study revealed that the desire to have heavier bodies among boys from LMICs backgrounds, reported in our cross-sectional study, may be because of common perception among mothers and their children that extra weight transforms to height. Due to only three girls in our qualitative sample, the body image preference was unclear among them, however, all three girls wanted to be taller. These qualitative results show a strong intergeneration cultural influence of origin countries, where the health promotion of governments is focused on weight gain to reduce undernutrition and stunting (Popkin, Adair, \& Ng, 2012; Williams, Crockett, Harrison, \& Thomas, 2012). The contradictions in weight preferences between origin and host countries, was perplexing for children. Children were encouraged to eat more to be stronger and taller at home by their mothers, but they were simultaneously conscious of the stigma, unpopularity, and low status associated with high bodyweights amongst their peers.

The intergenerational conflicts, and contradictions among immigrant mothers and their children identified in our qualitative study were also reported earlier (Olvera et al., 2005; Renzaho, McCabe, \& Swinburn, 2012; Rhodes et al., 2016). Through the maternal and childrens' accounts, we found that mothers prioritised and drew upon views about body image standards from their origin culture, but the children followed Australian norms. There was a disconnect with maternal awareness of obesity and adverse health effects and her desire for heavier bodyweights for her children.

Woven throughout these cultural contradictions regarding the meaning of weight, and body size were issues of gender and power. Girls from LMICs backgrounds showed higher BID and wanted to be thinner, although they did not have any significant weight difference from girls of other groups. These immigrant girls faced double jeopardy. If they were from countries which favour corpulence in girls (for example, certain African countries), they may resist parental pressures to gain weight inorder to be popular with their Australian peers (Renzaho, McCabe, \& Swinburn, 2012), causing interfamilial stress. However, if they were from countries which prefer thinness, such as South Asian countries included in the qualitative component of our study, they may be under even greater pressure to maintain a thin body; at home by parents and outside by peers. Pre-pubertal normal developmental changes may further compound BID for these girls (Jongenelis, Byrne, \& Pettigrew, 2014). So for girls in general and girls of immigrants in particular, multiple, interconnected social categories (such as immigrant status, gender, race, culture, language) seem to intersect and increase weight stigma (Himmelstein, Puhl, \& Quinn, 2017). Maternal and children's interviews reveal some of the mechanisms that were creating this double jeopardy for girls, for example, restrictions on physical activities for girls due to their cultural and religious norms (Renzaho, Green, Smith, \& Polonsky, 2017; Van Hook \& Baker, 2010). Successful obesity prevention intervention must include measures to address such barriers to physical activities with a special focus on immigrant girls. 
Similar to previous evidence, we found that home and school environment are strong determinants of health behaviours including food choices and dietary and physical activity practices for children (Larsen et al., 2015; Popovic-Lipovac \& Strasser, 2015; Wilson \& Renzaho, 2015). However, for immigrant children, inconsistencies between home and school environments increased their overweight/obesity risks, due to different standards of food choices, the level of physical activity and body image perception (Gualdi-Russo et al., 2014; Renzaho, McCabe, \& Swinburn, 2012). These results call for more research on intergeneration differences in food choices and dietary behaviours, body image standards and activity levels among immigrants.

The challenge for public health professionals is to craft health messages that are culturally appropriate and feasible for immigrant families. The findings of our mixed-method study show the complexity, challenges and need for dialogue with parents and children for cultural and gender-specific strategies. Although Australian health promotion raised maternal and child obesity awareness to some extent, it seems insufficient to guide healthy practices for obesity prevention. Health promotion activities focused on promoting positive behaviours such as information on how to prepare healthy home-cooked traditional meals may be more helpful for immigrants.

Finally, while mothers and children in our qualitative study were well informed about foods associated with overweight/obesity, fewer understood the need for physical activities. Given that many immigrants arrive from countries where sedentary activities are associated with high status (Renzaho, Green, Smith, \& Polonsky, 2017), increasing awareness about the benefits of physical activity in combating overweight/obesity alongside providing safe and culturally appropriate venues for physical activities are important.

\subsection{Strengths and Limitations}

To the best of our knowledge, this is the first mixed-methods Australian study which analysed a nationally representative cross-sectional dataset of 10-11-year-old children by their immigrant status and substantiated the quantitative results by narratives of mothers from three historically, linguistically, culinary and culturally similar LMICs and their children. Mixed-methods studies are quite challenging to analyse and are not frequently reported in research. However, adopting a mixed-methods design provided us with in-depth information about maternal and child perspectives on the trends observed in our cross-sectional study. Other strengths of our study included anthropometric measurements by trained interviewers for the cross-sectional study and use of the same body image instrument and questions to gauge body images in the cross-sectional and qualitative study. Also important was adding children own views to this discussion of BID and weight management alongside mothers to tease out intergenerational differences.

Sample weights were used to adjust for unequal probabilities of sample selection and non-response in our cross-sectional analyses, as the LSAC data underrepresents children from non-English speaking, single-parent families living in disadvantaged areas, and over-represents mothers with year 12 education. We also acknowledge that classification of maternal immigrant status by economic development of origin countries may have obscured some information on country specific cultural practices in the cross-sectional study.

Our qualitative study only included mothers from three LMICs with similar socio-economic and cultural backgrounds, which restrict generalisability of the results to immigrants from other countries. Our qualitative sample of mothers and their children was small. Further, the mothers belonged to middle income class in their origin countries and in Australia, and may be different than mothers from other other SES classes. Despite these limitations, the results of our qualitative study, affirmed findings from other studies on immigrant's health, which shows that similar pathways may exist amongst immigrants which increase the risk of overweight/obesity. The snowball sampling technique may also have introduced selection bias; therefore, caution is advised when interpreting the results for immigrants from less educated and low socioeconomic class.

Due to even smaller number of girls in our qualitative study, it was not possible to explain the gender-specific trends observed in the quantitative component. However, maternal interviews provided significant insight into how gender bias and culture intersect with regard to daily lives of boys and girls. Larger sample research is required to investigate these trends amongst children from other immigrant groups.

\section{Conclusions}

In conclusion, the results of the present study illustrate the challenges faced by immigrants and their children settling in countries with conflicting cultural norms of food, physical activities and body weight. The culture of origin countries has a strong role in what immigrants and their children eat, what activities they participate in and how they perceive their body shape. Children of immigrants may feel more conflicted, due to their desire to fit in the host society but may also be pressured to keep their parental cultural identities intact. Girls of immigrants from LMICs are further disadvantaged due to cultural, religious and other complex intersectional and intergenerational 
differences.

Our study revealed intergenerational dilemmas for the mothers and the children. Mothers reported pressures on them and their parenting from their friends and relatives in their host countries as well as in origin countries. The children, on the other hand, reported different norms and standards and peer pressure creating confusion and presenting dilemmas between the mothers and their children. Our results indicate the complexity of addressing health issues amongst immigrant generations. It is important for public health interventions to be culturally respectful, but at the same time empower immigrant mothers to understand weight and activities in different ways.

At the time when Australia is developing a national obesity strategy, it is important to be aware of the disadvantages faced by children of immigrants. High awareness of health messages on dietary causes of obesity and low awareness about physical activities reported in this study indicate that culturally appropriate and feasible physical activity messages are required which are acceptable to the immigrants. Our study shows that for children a primary developmental need is to connect to their peers and be accepted and valued. It is complicated for these children because of their culturally dissimilar backgrounds and therefore fitting in is even a bigger challenge. Our results identify a challenge for public health to develop interventions that grapple with gender, culture and status related meanings of food, physical activities, and body images in a way that do not undermine cultural identities, yet help these families understand healthy weights.

\section{Competing Interests Statement}

The authors declare that there are no competing or potential conflicts of interest.

\section{References}

Australian Bureau of statistics. (2012). Cultural Diversity in Australia; Reflecting a Nation: Stories from the 2011 Census, 2012-2013. http://www.abs.gov.au/ausstats/abs@.nsf/Lookup/2071.0main+features902012-2013

Australian Bureau of statistics. (2015). National Health Survey: First Results, 2014-15. http://www.abs.gov.au/ausstats/abs@.nsf/mf/4364.0.55.001

Australian Institute of Family Studies. (2014). The Longitudinal Study of Australian Children Annual statistical report 2013. Melbourne; Australia.

Australian Institute of Health and Welfare. (2018). Culturally and linguistically diverse populations. AIHW; Canberra: Australia.

Baker, E., Balistreri, K. S., \& Van Hook, J. (2009). Maternal Employment and Overweight Among Hispanic Children of Immigrants and Children of Natives. Journal of Immigrant and Minority Health, 11(3), 158-167. https://doi.org/10.1007/s10903-007-9096-0

Birbeck, D., \& Drummond, M. (2005). Interviewing, and listening to the voices of, very young children on body image and perceptions of self. Early Child Development and Care, 175(6), 579-596. https://doi.org/10.1080/03004430500131379

Blakemore, T., Strazdins, L., \& Gibbings, J. (2009). Measuring family socioeconomic position. Australian Social Policy, 8(121-168), 121-168.

Braun, V., Clarke, V., \& Terry, G. (2014). Thematic analysis. Qual Res Clin Health Psychol, 24, 95-114. https://doi.org/10.1037/13620-004

Cole, T. J., Bellizzi, M. C., Flegal, K. M., \& Dietz, W. H. (2000). Establishing a standard definition for child overweight and obesity worldwide: international survey. $B M J, 320(7244), 1240$. https://doi.org/10.1136/bmj.320.7244.1240

Collins, M. E. (1991). Body figure perceptions and preferences among preadolescent children. International $\begin{array}{lllll}\text { Journal of } & \text { Eating } & \text { Disorders, } & 10(2), & \text { 199-208. }\end{array}$ https://doi.org/10.1002/1098-108X(199103)10:2<199::AID-EAT2260100209>3.0.CO;2-D

Daraganova, G. (2013). Body image of primary school children. LSAC Annual statistical report 2013. Melbourne: Australia.

Gibson, L. Y., Allen, K. L., Byrne, S. M., Clark, K., Blair, E., Davis, E., \& Zubrick, S. R. (2016). Childhood Overweight and Obesity: Maternal and Family Factors. Journal of Child and Family Studies, 1-11. https://doi.org/10.1007/s10826-016-0485-7

Gualdi-Russo, E., Manzon, V. S., Masotti, S., Toselli, S., Albertini, A., Celenza, F., \& Zaccagni, L. (2012). Weight status and perception of body image in children: the effect of maternal immigrant status. Nutrition journal, 11, 
85. https://doi.org/10.1186/1475-2891-11-85

Gualdi-Russo, E., Zaccagni, L., Manzon, V. S., Masotti, S., Rinaldo, N., \& Khyatti, M. (2014). Obesity and physical activity in children of immigrants. European Journal of Public Health, 24(Suppl 1), 40-46. https://doi.org/10.1093/eurpub/cku111

Hancock, K. J., Lawrence, D., \& Zubrick, S. R. (2014). Higher maternal protectiveness is associated with higher odds of child overweight and obesity: a longitudinal Australian study. PloS one, 9(6), e100686. https://doi.org/10.1371/journal.pone.0100686

Himmelstein, M. S., Puhl, R. M., \& Quinn, D. M. (2017). Intersectionality: an understudied framework for addressing weight stigma. American journal of preventive medicine, 53(4), 421-431. https://doi.org/10.1016/j.amepre.2017.04.003

Jones, D. C., Bain, N., \& King, S. (2008). Weight and muscularity concerns as longitudinal predictors of body image among early adolescent boys: A test of the dual pathways model. Body Image, 5(2), 195-204. https://doi.org/10.1016/j.bodyim.2007.12.001

Jongenelis, M. I., Byrne, S. M., \& Pettigrew, S. (2014). Self-objectification, body image disturbance, and eating disorder symptoms in young Australian children. Body Image, 11(3), $290-302$. https://doi.org/10.1016/j.bodyim.2014.04.002

Kimber, M., Couturier, J., Georgiades, K., Wahoush, O., \& Jack, S. M. (2015). Ethnic Minority Status and Body Image Dissatisfaction: A Scoping Review of the Child and Adolescent Literature. Journal of Immigrant and Minority Health, 17(5), 1567-1579. https://doi.org/10.1007/s10903-014-0082-z

Klein, J. S. (2008). Body image in boys: the role of race, positive affect and peer popularity in eighth grade boys. Columbia University.

Larsen, J. K., Hermans, R. C., Sleddens, E. F., Engels, R. C., Fisher, J. O., \& Kremers, S. S. (2015). How parental dietary behavior and food parenting practices affect children's dietary behavior. Interacting sources of influence? Appetite, 89, 246-257. https://doi.org/10.1016/j.appet.2015.02.012

Linabery, A. M., Nahhas, R. W., Johnson, W., Choh, A. C., Towne, B., Odegaard, A. O., . . Demerath, E. W. (2013). Stronger influence of maternal than paternal obesity on infant and early childhood body mass index: the Fels Longitudinal Study. Pediatric obesity, $8(3), \quad$ 159-169. https://doi.org/10.1111/j.2047-6310.2012.00100.x

Mitchison, D., \& Mond, J. (2015). Epidemiology of eating disorders, eating disordered behaviour, and body image disturbance in males: a narrative review. Journal of eating disorders, 3(1), 20. https://doi.org/10.1186/s40337-015-0058-y

Mond, J., Hall, A., Bentley, C., Harrison, C., Gratwick-Sarll, K., \& Lewis, V. (2014). Eating-disordered behavior in adolescent boys: Eating disorder examination questionnaire norms. International Journal of Eating Disorders, 47(4), 335-341. https://doi.org/10.1002/eat.22237

O'dea, J. A., \& Caputi, P. (2001). Association between socioeconomic status, weight, age and gender, and the body image and weight control practices of 6-to 19-year-old children and adolescents. Health education research, 16(5), 521-532. https://doi.org/10.1093/her/16.5.521

Olvera, N., Suminski, R., \& Power, T. G. (2005). Intergenerational perceptions of body image in Hispanics: Role of BMI, gender, and acculturation. Obesity research, 13(11), 1970-1979. https://doi.org/10.1038/oby.2005.242

Pallan, M. J., Hiam, L. C., Duda, J. L., \& Adab, P. (2011). Body image, body dissatisfaction and weight status in south asian children: a cross-sectional study. BMC Public Health, 11(1), 21. https://doi.org/10.1186/1471-2458-11-21

Park, S. H. (2017). Asian Parents' Perception of Child Weight Status: A Systematic Review. Journal of Child and Family Studies, 26(9), 2363-2373. https://doi.org/10.1007/s10826-017-0765-X

Pasupuleti, S. S. R., Jatrana, S., \& Richardson, K. (2016). Effect of nativity and duration of residence on chronic health conditions among Asian immigrants in Australia: a longitudinal investigation. Journal of biosocial science, 48(3), 322-341. https://doi.org/10.1017/S0021932015000206

Popkin, B. M., Adair, L. S., \& Ng, S. W. (2012). Now and Then: The Global Nutrition Transition: The Pandemic of Obesity in Developing Countries. Nutrition Reviews, 70(1), 3-21. 
https://doi.org/10.1111/j.1753-4887.2011.00456.x

Popovic-Lipovac, A., \& Strasser, B. (2015). A review on changes in food habits among immigrant women and implications for health. Journal of immigrant minority health, 17(2), 582-590. https://doi.org/10.1007/s10903-013-9877-6

Renzaho, A. M., Green, J., Smith, B. J., \& Polonsky, M. (2017). Exploring Factors Influencing Childhood Obesity Prevention Among Migrant Communities in Victoria, Australia: A Qualitative Study. Journal of immigrant and minority health, 1-19. https://doi.org/10.1007/s10903-017-0620-6

Renzaho, A. M., McCabe, M., \& Swinburn, B. (2012). Intergenerational differences in food, physical activity, and body size perceptions among African migrants. Qual Health Res, 22(6), 740-754. https://doi.org/10.1177/1049732311425051

Rhodes, K., Chan, F., Prichard, I., Coveney, J., Ward, P., \& Wilson, C. (2016). Intergenerational transmission of dietary behaviours: a qualitative study of Anglo-Australian, Chinese-Australian and Italian-Australian three-generation families. Appetite, 103, 309-317. https://doi.org/10.1016/j.appet.2016.04.036

Ricciardelli, L. A., \& McCabe, M. P. (2001). Children's body image concerns and eating disturbance: A review of the literature. Clinical psychology review, 21(3), 325-344. https://doi.org/10.1016/S0272-7358(99)00051-3

Ricciardelli, L. A., McCabe, M. P., Holt, K. E., \& Finemore, J. (2003). A biopsychosocial model for understanding body image and body change strategies among children. Journal of Applied Developmental Psychology, 24(4), 475-495. https://doi.org/10.1016/S0193-3973(03)00070-4

Rosen, J. C. (2013). Body image disorder: Definition, development, and contribution to eating disorders. The etiology of bulimia nervosa: The individual and familial context, 1, 157-177.

Sadler, G. R., Lee, H. C., Lim, R. S. H., \& Fullerton, J. (2010). Recruitment of hard-to-reach population subgroups via adaptations of the snowball sampling strategy. Nursing \& health sciences, 12(3), 369-374. https://doi.org/10.1111/j.1442-2018.2010.00541.x

Saldaña, J. (2015). The coding manual for qualitative researchers. USA. Sage publications.

Smolak, L. (2004). Body image in children and adolescents: where do we go from here? Body image, 1(1), 15-28. https://doi.org/10.1016/S1740-1445(03)00008-1

Soloff, C., Lawrence, D., \& Johnstone, R. (2005). LSAC technical paper no. 1: Sample design. Melbourne. Australia: Australian Institute of Family Studies.

Toselli, S., Brasili, P., \& Spiga, F. (2014). Body image, body dissatisfaction and weight status in children from Emilia-Romagna (Italy): comparison between immigrant and native-born. Annals of human biology, 41(1), 23-28. https://doi.org/10.3109/03014460.2013.822557

Tracy, S. J. (2013). Qualitative research methods. UK: Wiley-Blackwell.

UNDP. (2016). Human development for everyone, Human development report 2016. United Nations, New York.

Van Griensven, H., Moore, A. P., \& Hall, V. (2014). Mixed methods research-The best of both worlds? Manual therapy, 19(5), 367-371. https://doi.org/10.1016/j.math.2014.05.005

Van Hook, J., \& Baker, E. (2010). Big Boys and Little Girls: Gender, Acculturation, and Weight among Young Children of Immigrants. Journal of health and social behavior, 51(2), 200-214. https://doi.org/10.1177/0022146510372347

Veldhuis, J., te Poel, F., Pepping, R., Konijn, E. A., \& Spekman, M. L. (2017). "Skinny is prettier and normal: I want to be normal"-Perceived body image of non-Western ethnic minority children in the Netherlands. Body image, 20, 74-86. https://doi.org/10.1016/j.bodyim.2016.11.006

WHO Expert Consultation. (2004). Appropriate body-mass index for Asian populations and its implications for policy and intervention strategies. Lancet (London, England), 363(9403), 157.

Williams, J. D., Crockett, D., Harrison, R. L., \& Thomas, K. D. (2012). The role of food culture and marketing activity in health disparities. Preventive Medicine, 55(5), 382-386. https://doi.org/10.1016/j.ypmed.2011.12.021

Wilson, A., \& Renzaho, A. (2015). Intergenerational differences in acculturation experiences, food beliefs and perceived health risks among refugees from the Horn of Africa in Melbourne, Australia. Public health nutrition, 18(1), 176-188. https://doi.org/10.1017/S1368980013003467 
Ziauddeen, N., Roderick, P. J., Macklon, N. S., \& Alwan, N. A. (2018). Predicting childhood overweight and obesity using maternal and early life risk factors: a systematic review. Obesity Reviews, 19(3), $302-312$. https://doi.org/10.1111/obr.12640

Zulfiqar, T., Strazdins, L., Banwell, C., Dinh, H., \& D'Este, C. (2018). Growing up in Australia: paradox of overweight/obesity in children of immigrants from low-and-middle-income countries. Obesity science \& practice, 4(2), 178-187. https://doi.org/10.1002/osp4.160

Zulfiqar, T., Strazdins, L., Dinh, H., Banwell, C., \& D’Este, C. (2018). Drivers of Overweight/Obesity in 411-Year-Old Children of Australians and Immigrants; Evidence from Growing Up in Australia. Journal of immigrant and minority health, 1-14. https://doi.org/10.1007/s10903-018-0841-3

\section{Supplementary tables}

Table 1S. Body image dissatisfaction in 10-11-year-old boys and girls by their immigrant status

\begin{tabular}{|c|c|c|c|c|c|}
\hline & $\begin{array}{l}\text { Satisfied with } \\
\text { body image } \\
\mathrm{n}(\%)\end{array}$ & $\begin{array}{l}\text { Unsatisfied and want heavy } \\
\text { body image } \\
\mathrm{n}(\%)\end{array}$ & $\begin{array}{l}\text { Unsatisfied and want thin } \\
\text { body image } \\
\mathrm{n}(\%)\end{array}$ & Total & $\begin{array}{l}\mathrm{P} \\
\text { value }\end{array}$ \\
\hline Australian boys & $828(55.1)$ & $115(8.7)$ & $504(36.2)$ & 1447 & \multirow{3}{*}{0.002} \\
\hline $\begin{array}{l}\text { Boys with HICs } \\
\text { backgrounds }\end{array}$ & $127(67.2)$ & $8(3.5)$ & $57(29.3)$ & 192 & \\
\hline $\begin{array}{l}\text { Boys with LMICs } \\
\text { backgrounds }\end{array}$ & $83(48.2)$ & 57 (13.9) & $65(37.8)$ & 170 & \\
\hline Australian girls & $783(54.6)$ & $65(5.1)$ & $555(40.3)$ & 1403 & \multirow{3}{*}{0.008} \\
\hline $\begin{array}{l}\text { Girls with HICs } \\
\text { backgrounds }\end{array}$ & $109(61.0)$ & $16(8.0)$ & $56(30.9)$ & 181 & \\
\hline $\begin{array}{l}\text { Girls with LMICs } \\
\text { backgrounds }\end{array}$ & $66(43.5)$ & $15(8.1)$ & $75(48.4)$ & 156 & \\
\hline
\end{tabular}

- Abbreviations: HICs=high-income-countries, LMICs= Low-and-middle-income-countries

- Percentages are weighted and rounded.

- P-value for chi-square test comparing variables across immigrant status (Australian, HICs \& LMICs).

- Numbers may not add due to missing values 
Table 2S. Percentage of 10-11-year-old boys and girls who adopted weight management strategies in B Cohort of longitudinal study of Australian Children in the previous 12 months

\begin{tabular}{|c|c|c|c|c|c|c|}
\hline & Lose weight & Gain weight & Stay the same & not doing any thing & Total & $\mathrm{P}$ value \\
\hline Australian boys & $553(40.1)$ & $84(5.9)$ & $516(33.9)$ & $294(20.2)$ & 1447 & \\
\hline Boys with HICs backgrounds & $73(40.9)$ & $12(5.9)$ & $67(34.4)$ & $40(17.7)$ & 192 & $<0.001$ \\
\hline Boys with LMICs backgrounds & $76(45.7)$ & $27(17.7)$ & $39(20.9)$ & $30(15.7)$ & 172 & \\
\hline Australian girls & $548(40.8)$ & $33(2.6)$ & $430(30.6)$ & $389(25.9)$ & 1400 & \\
\hline Girls with HICs backgrounds & $48(27.8)$ & $10(4.3)$ & $61(33.2)$ & $62(34.7)$ & 181 & $<0.001$ \\
\hline Girls with LMICs backgrounds & $71(45.4)$ & $13(10.3)$ & $38(25.9)$ & $34(19.4)$ & 156 & \\
\hline
\end{tabular}

- Abbreviations: HICs=high-income-countries, LMICs= Low-and-middle-income-countries

- Percentages are weighted and rounded.

- P-value for chi-square test comparing variables across immigrant status (Australian, HICs \& LMICs).

- Numbers may not add due to missing values

\section{Copyrights}

Copyright for this article is retained by the author(s), with first publication rights granted to the journal.

This is an open-access article distributed under the terms and conditions of the Creative Commons Attribution license (http://creativecommons.org/licenses/by/4.0/). 